\title{
Spontaneous Coronary Artery Dissection: The Phantom Menace
}

\author{
Nikolaos Spinthakis ${ }^{\mathrm{a}, \mathrm{b}}$, Nada Abdulkareem ${ }^{\mathrm{a}}$, Mohamed Faraga, b, Diana A. Gorog a, b, c, d
}

\begin{abstract}
We present a case of a 66-year-old lady with chest pain, without dynamic 12-lead electrocardiographic (ECG) changes and normal serial troponin. Coronary angiography revealed a linear filing defect in the first obtuse marginal branch of the circumflex artery indicating coronary artery dissection, with superadded thrombus. She was managed medically with dual antiplatelet therapy and has responded well. Spontaneous coronary artery dissection (SCAD) is a rare cause of cardiac chest pain, which can be missed without coronary angiography. Unlike most other lesions in patients with unstable symptoms, where coronary intervention with stenting is recommended, patients with SCAD generally fare better with conservative measures than with intervention, unless there is hemodynamic instability.
\end{abstract}

Keywords: Coronary artery; Dissection; Phantom menace; Conservative measure

\section{Introduction}

Spontaneous coronary artery dissection (SCAD) is a rare and underdiagnosed cause of acute coronary syndrome (ACS) with a prevalence of $0.07-1.1 \%$ [1]. It predominantly affects women, with a peak incidence in the fifth decade, and more often during the peripartum period. It can occur with or without coronary atheroma. The cause of SCAD remains unclear with cases being referred as idiopathic, when atherosclerotic disease is not present, predominantly affecting female patients in multiple coronary sites [1]. Most commonly SCAD presents with intimal-media tear, resulting in a communication between the vessel lumen and the intramural hematoma, which can be visualized as a filing defect at coronary angiography. On the other hand, there have been several reported cases where an intramural hematoma is formed, leading to narrowing of the true

Manuscript accepted for publication December 12, 2016

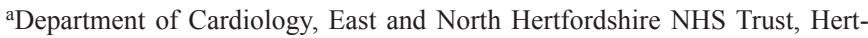
fordshire, UK

bPostgraduate Medicine, The University of Hertfordshire, UK

'National Heart \& Lung Institute, Imperial College, London, UK

${ }^{\mathrm{d} C}$ Corresponding Author: Diana A. Gorog, Imperial College, London, UK.

Email: d.gorog@imperial.ac.uk

doi: https://doi.org/10.14740/cr513w lumen [2]. Such cases where angiography does not reveal an intraluminal filling defect may be detected with intravascular ultrasound (IVUS) and coronary computed tomographic angiography (CCTA) [3-5]. However, this option has sometimes led to over-aggressively treating the dissection with coronary intervention, often leading to non-desirable outcomes. In particular if the dissection affects small vessels or distal areas, multiple wiring maneuvers or strong contrast injections may lead to progression of the dissection or even abrupt vessel closure [6]. Lack of universal consensus in the management of SCAD commonly leads to a case-based approach relying on clinical and radiological findings. Conservative medical management with the addition of watchful waiting has been shown to have the most favorable outcome in clinically stable patients. Coronary angioplasty should be reserved for dissections with extension or unstable condition [1]. Finally coronary artery bypass surgery may be reserved for unstable cases with unfavorable anatomy for percutaneous coronary intervention (PCI) [1].

\section{Case Report}

A 66-year-old woman presented to the emergency department with central crushing chest pain radiating to her left arm associated with breathlessness. She had chest pain 3 years ago and had previously been investigated with a coronary angiogram, which had revealed unobstructed coronaries, with mild atheroma only in the left anterior descending artery (LAD) (Fig. 1). Her past medical history was notable for type 2 diabetes mellitus, hyperlipidemia, essential hypertension, osteoarthritis and microcytic anemia. She denied any flu-like symptoms recently; she was a non-smoker, who had no intake of recreational drugs. Medication included aspirin $75 \mathrm{mg}$ od, bisoprolol 1.25 $\mathrm{mg}$ od, diltiazem $120 \mathrm{mg}$ tds, nicorandil $10 \mathrm{mg}$ bd, rosuvastatin $10 \mathrm{mg}$ od, omeprazole $20 \mathrm{mg}$ od, isosorbide mononitrate MR $120 \mathrm{mg}$ od, glyceryl trinitrate sublingual spray (GTN) as required, metformin $1 \mathrm{~g} \mathrm{bd}$, gliclazide $40 \mathrm{mg}$ od and ferrous fumarate $200 \mathrm{mg}$ bd. Examination was normal with a blood pressure of 130/74 $\mathrm{mm} \mathrm{Hg}$ and she was in sinus rhythm at 69 beats per minute with oxygen saturation of $98 \%$ on room air. Initial 12-lead electrocardiographic (ECG) was normal (Fig. 2 ) as were the following serial ECGs. Troponin measured $3 \mathrm{~h}$ after the onset of pain was $<3(0-14 \mathrm{ng} / \mathrm{L})$. Chest X-ray was normal and baseline blood tests were unremarkable with hemoglobin of $121 \mathrm{~g} / \mathrm{L}$ and admitting blood sugar levels of 10.7 $\mathrm{mmol} / \mathrm{L}$. GRACE score upon presentation was 95, placing her at moderate risk for major adverse cardiac events, and subsequently she was admitted to the acute cardiac unit for cardiac 


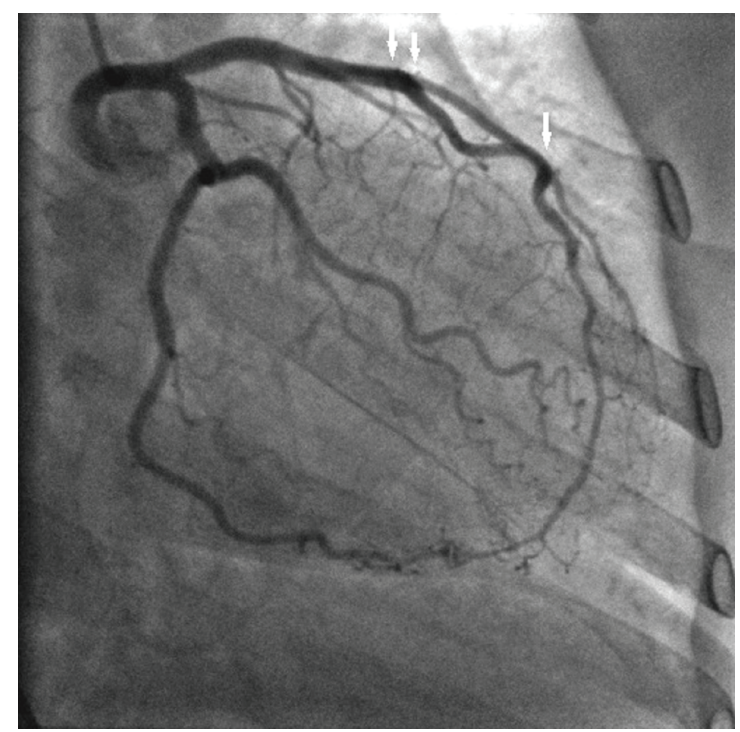

Figure 1. Coronary angiography showing mild atheroma in the LAD (white arrows).

monitoring. She was initially treated for a suspected ACS with a loading dose of aspirin, clopidogrel and fondaparinux, morphine analgesia and GTN spray. Unfortunately, the pain, which initially settled, recurred in a more severe manner and a decision was made to refer her for an urgent coronary angiogram.

\section{Investigations}

The 12-lead ECG was normal (Fig. 2) as were the serial ECGs. Troponin measured $3 \mathrm{~h}$ after the onset of pain was $<3(0-14$ $\mathrm{ng} / \mathrm{L}$ ), chest X-ray was normal and baseline blood tests were

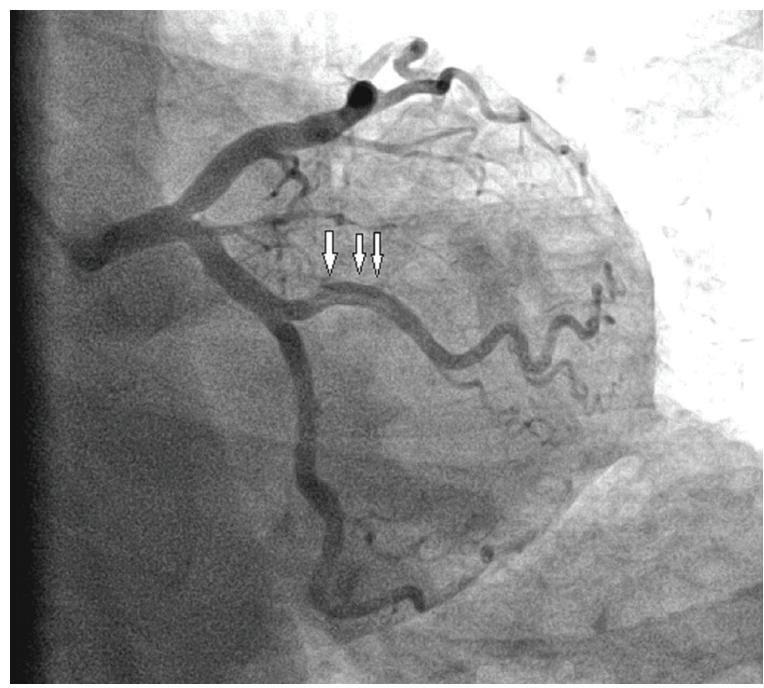

Figure 3. Coronary angiography revealed a linear filing defect with an overlying thrombus in the first obtuse marginal branch of the circumflex coronary artery (white arrows) suggestive of SCAD.

unremarkable with a hemoglobin of $121 \mathrm{~g} / \mathrm{L}$ and admitting blood sugar levels of $10.7 \mathrm{mmol} / \mathrm{l}$.

Coronary angiography revealed a linear filing defect with an overlying thrombus in the first obtuse marginal branch of the circumflex coronary artery, SCAD (Fig. 3), with otherwise unobstructed coronaries.

\section{Differential diagnoses}

Differential diagnoses included ACS (unstable angina/non-ST elevation myocardial infarction), coronary artery spasm, and

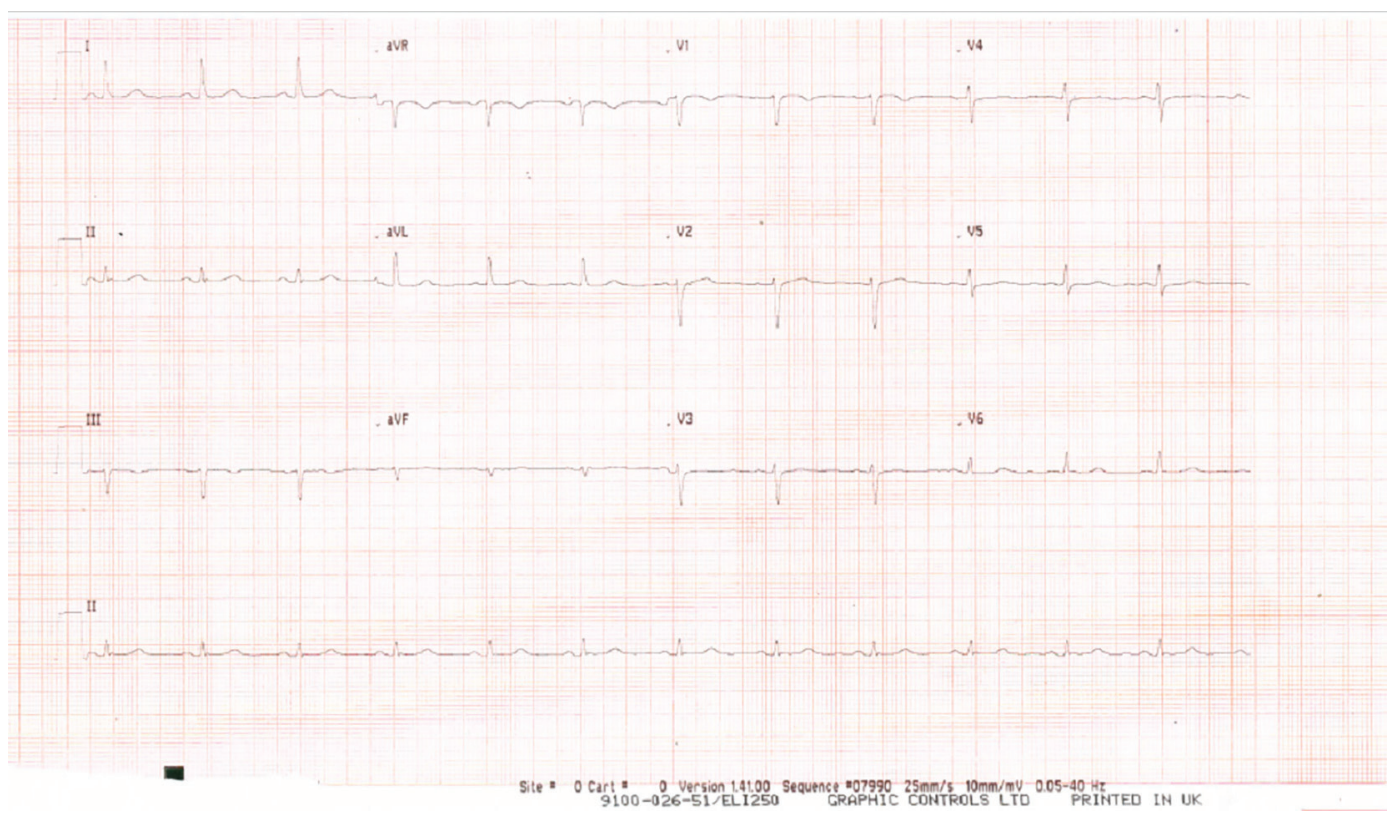

Figure 2. Normal 12-lead ECG on presentation. 
non-cardiac causes of chest pain including musculoskeletal pain.

\section{Treatment}

She was initially treated for suspected ACS with a loading dose of aspirin and clopidogrel and fondaparinux. She was hemodynamically stable and a decision was made to continue with conservative medical management with dual antiplatelet therapy.

\section{Outcome and follow-up}

During the admission, she remained well with no complications and was discharged on day 3 on dual antiplatelet therapy, with aspirin $75 \mathrm{mg}$ and clopidogrel $75 \mathrm{mg}$ for 1 year in addition to her medication and thereon aspirin monotherapy. Bisoprolol was increased to $7.5 \mathrm{mg}$ od, rosuvastatin was changed to atorvastatin $40 \mathrm{mg}$ od and diltiazem was discontinued. A year later, she remains well and is completely asymptomatic.

\section{Discussion}

SCAD is a rare cause of angina and a challenging diagnosis to make, requiring high degree of suspicion. Our understanding of SCAD comes from case series, case reports and small single center registries. These indicate that SCAD most commonly affects single vessels (and most commonly the LAD) in both men and women, and most commonly presents initially with ACS [1]. Interestingly, the prevalence of SCAD may be underappreciated, as often it is associated with sudden cardiac death [7]. Saw has previously classified SCAD into different types based on angiographic findings and highlighted the importance of the invisible dissection, suggesting the use of other imaging modalities such as IVUS and optical coherence tomography (OCT) [2]. When dissection is suspected in the absence of significant coronary artery disease in the context of ACS, IVUS and OCT may aid the diagnosis $[3-5,8]$. In terms of managing this condition, the lack of clinical trials has led physicians to apply a case-based clinical and imaging risk-stratification model. Invasive management with PCI has shown to be beneficial in hemodynamically unstable cases and coronary artery bypass graft (CABG) surgery can be considered for multi-vessel involvement or when anatomy is unfavorable for PCI [1, 9]. In stable patients with single vessel disease, the evidence suggests a more favorable outcome with medical management and close surveillance than with coronary intervention $[7,10]$. In a recent contemporary review of the literature, Saw et al suggested a conservative approach to be superior to intervention as spontaneously arterial healing occurs more frequently, which avoids the risks of revascularization and risk of restenosis [9]. Alfonso et al in a case series of 45 consecutive patients with SCAD showed a relatively low rate of recurrent major adverse cardiac events $(7 \%)$ over a follow-up period of 2 years with a conservative approach [10]. Another interest- ing point noted, is the rate of spontaneous arterial healing with medical management, which has been observed in three large case series at a rate of $85 \%$ on repeat angiography [9]. Resorption of the dissection seems to occur immediately; however, it may take up to 26 days to heal completely [9]. In conclusion, awareness is required in order to identify SCAD early and conservative treatment with close follow-up should be recommended, unless there are hemodynamic factors or reduced antegrade coronary flow.

\section{Learning points}

High degree of suspicion is required in order to identify SCAD. Coronary angiography remains the gold standard in diagnosis; however, use of IVUS, OCT, and CCTA should be considered to assist diagnosis when angiography does not reveal dissection and there is high clinical suspicion.

Conservative medical management with dual antiplatelet therapy should be considered for hemodynamically stable patients.

\section{References}

1. Giacoppo D, Capodanno D, Dangas G, Tamburino C. Spontaneous coronary artery dissection. Int J Cardiol. 2014;175(1):8-20.

2. Saw J. Coronary angiogram classification of spontaneous coronary artery dissection. Catheter Cardiovasc Interv. 2014;84(7):1115-1122.

3. Buccheri D, Piraino D, Andolina G. Multimodality imaging for spontaneous coronary artery dissection: A clinical points system may be a shortcut to diagnosis. Int J Cardiol. 2016;214:391-392.

4. Arnold JR, West NE, van Gaal WJ, Karamitsos TD, Banning AP. The role of intravascular ultrasound in the management of spontaneous coronary artery dissection. Cardiovasc Ultrasound. 2008;6:24.

5. Maehara A, Mintz GS, Castagna MT, Pichard AD, Satler LF, Waksman R, Suddath WO, et al. Intravascular ultrasound assessment of spontaneous coronary artery dissection. Am J Cardiol. 2002;89(4):466-468.

6. Sanz-Ruiz R, Diez-Delhoyo F, Fernandez-Aviles F. Further insights on spontaneous coronary artery dissection: Scores, imaging and optimal management. Int J Cardiol. 2016;222:997-998.

7. Tweet MS, Hayes SN, Pitta SR, Simari RD, Lerman A, Lennon RJ, Gersh BJ, et al. Clinical features, management, and prognosis of spontaneous coronary artery dissection. Circulation. 2012;126(5):579-588.

8. Saw J, Mancini GB, Humphries K, Fung A, Boone R, Starovoytov A, Aymong E. Angiographic appearance of spontaneous coronary artery dissection with intramural hematoma proven on intracoronary imaging. Catheter Cardiovasc Interv. 2016;87(2):E54-61.

9. Saw J, Mancini GB, Humphries KH. Contemporary Review on Spontaneous Coronary Artery Dissection. J Am Coll Cardiol. 2016;68(3):297-312. 
10. Alfonso F, Paulo M, Lennie V, Dutary J, Bernardo E, Jimenez-Quevedo P, Gonzalo N, et al. Spontaneous coronary artery dissection: long-term follow-up of a large series of patients prospectively managed with a "conservative" therapeutic strategy. JACC Cardiovasc Interv. 2012;5(10):1062-1070. 